\title{
Evolving concepts of glycogen-selective autophagy
}

Parisa Koutsifeli ${ }^{1,2}$, Upasna Varma ${ }^{2}$, Lorna J. Daniels ${ }^{1,3}$, Marco Annandale ${ }^{1}$, Xun Li $^{1}$, Sarah Hayes ${ }^{2}$, Samuel James ${ }^{1}$, Lea M.D. Delbridge ${ }^{2,1}$, Kimberley M. Mellor ${ }^{1,2,4}$

${ }^{1}$ Department of Physiology, University of Auckland, New Zealand

${ }^{2}$ Department of Physiology, University of Melbourne, Australia

${ }^{3}$ Oxford Centre for Diabetes, Endocrinology and Metabolism, Radcliffe Department of Medicine, University of Oxford, Oxford, UK

${ }^{4}$ Auckland Bioengineering Institute, University of Auckland, New Zealand

Keywords (5): autophagy, glycogen, lysosome, glycophagy, Atg8, Stbd1, Gabarap11

\section{Corresponding Authors:}

Dr Kim M Mellor

Cellular and Molecular Cardiology Laboratory

Department of Physiology,

University of Auckland, New Zealand

k.mellor@auckland.ac.nz

Prof Lea MD Delbridge

Cardiac Phenomics Laboratory

Department of Physiology,

University of Melbourne, Australia.

$\underline{\text { lmd@unimelb.edu.au }}$ 


\begin{abstract}
Macro-autophagy is an essential cellular process involved in degradation of aberrant organelles and proteins. Initially proposed to be a 'bulk' degradation pathway, a more nuanced appreciation of selective autophagy pathways has emerged in recent years. The discovery of a glycogen-selective autophagy pathway ('glycophagy') has highlighted the importance of autophagy in regulating cellular metabolic homeostasis and identified a novel non-canonical major pathway of glycogen flux. The field of glycogen autophagy research is at an early evolutionary stage, but already it is clear that the implications of these discoveries are far-reaching and provide scope for multidisciplinary investigations into the role of glycophagy in health and disease. With potential cognate protein partners identified, the opportunities for targeted intervention have become viable. Here we review the current evidence relating to specific protein mediators involved in glycophagy, and highlight areas of uncertainty that provide opportunity for further investigation.
\end{abstract}




\section{Introduction}

Macro-autophagy (from the Greek 'self-eating') is an essential cellular process that engulfs cytoplasmic material into autophagosomes, delivering it to lysosomes for degradation (1). Although autophagy was initially characterized as a non-selective 'bulk' degradation process, more recently the concept of selective-autophagy has emerged, with specific protein mediators targeting organelles and macromolecules for destruction $(2,3)$. Several selective-autophagy pathways have been identified which target mitochondria (mitophagy), endoplasmic reticulum (ER-phagy), lipids (lipophagy) and glycogen (glycophagy) $(4,5)$. Although lysosomal degradation of glycogen has been anecdotally reported for several decades, only recently has the concept of glycophagy gained attention in the field. Glycophagy appears to operate in parallel with the well-described cytosolic glycogen breakdown pathway (involving glycogen phosphorylase) $(6,7)$. Important avenues of investigation are developing as new knowledge of this non-canonical pathway of glycogen flux is reported. This short review examines the current evidence for a glycogen-selective autophagy pathway. Literature related to identifying the distinct glycophagy molecular machinery is dissected, and important knowledge gaps are highlighted.

\section{An overview of glycogen-specific autophagy machinery}

An understanding of the unique protein intermediaries of glycophagy is emerging, and some insight into glycogen tagging and recruitment to the glyco-autophagosome is available from in vitro colocalization studies and proteomic analysis of glycogen-associated proteins. Further, inherited lysosomal diseases have been informative in relation to understanding the functional consequences of disturbances in lysosomal glycogen degradation. We have reported the first evidence indicating that glycophagy is operational in the adult heart, and is distinct from LC3-mediated macroautophagy $(8,9)$. As depicted in Figure 1 , the current state of knowledge relating to glycogenspecific autophagy protein machinery (primarily derived from in vitro cell line studies) implicates starch-binding domain-containing protein 1 (Stbd1) as the 'glycophagy receptor', tagging glycogen 
for autophagic degradation via its carbohydrate binding domain (CBM20) (10). Sequence data and co-localization studies support a role for Stbd1 recruiting glycogen to the forming phagosome via binding to the Atg8 'partner' protein, Gabarapl1 $(11,12)$. Following autophagosome-lysosome fusion, acid $\alpha$-glucosidase (Gaa) is the likely mediator of lysosomal glycogen breakdown $(13,14)$. We have shown that glycogen-specific autophagy machinery is responsive to insulin and glucose stimuli in vitro (8) and appears to be involved in cardiac glycogen homeostatic regulation in relation to metabolic stress in vivo (9). Thus characterization of some of the key players in the glycophagy machinery has been documented, but a full understanding of the pathway is limited, and significant knowledge gaps remain.

\section{Glycogen structure - a key determinant of glycophagy fate?}

With cytosolic (phosphorylase) and lysosomal (glycophagy) glycogenolysis pathways operating in parallel, it is not clear what determines the metabolic fate of glycogen. The precise regulatory mechanisms involved in targeting glycogen for glycophagy have not yet been defined, but some insight can be gained from reports identifying the glycogen structural characteristics most closely aligned with glycophagy occurrence. In the adult rat liver, at least $10 \%$ of cellular glycogen is confined to lysosome compartments, and lysosome-localized glycogen particles have a higher molecular weight than those in the cytosol (15). In the literature, glycogen has been categorized by both weight (low vs. high molecular weight) and size (small $40 \mathrm{~nm} \beta$-granules aggregate via disulfide bonds to form large $\sim 300 \mathrm{~nm} \alpha$-granules) $(6,7)$. Although not directly investigated, it could be expected that high molecular weight glycogen particles detected in the lysosome are consistent with large $\alpha$-granules. Further research is required to understand the implications of potential glycophagy 'preference’ for large protein-rich $\alpha$-granules. Given that $\alpha$-granules exhibit protein backbones and disulfide bonds linking smaller $\beta$-granules $(16,17)$, it could be postulated that the lysosomal environment is optimal for breaking disulfide linkages via lysosomal thiol reductase (18), 
in concert with lysosomal proteases degrading the glycogen-associated proteins, and glucosidases cleaving the glucose strands - a coordinated process not accessible to cytosolic glycogen.

There is some evidence to suggest that glycogen branching content may also determine glycophagy fate. The glycophagy-tagging 'receptor' protein, Stbd1, and lysosomal glucosidases exhibit a higher affinity for less branched polysaccharides in an experimental in vitro context using recombinant human proteins and polysaccharide extracts $(10,19)$. Further, glycophagy activity is highest in rats in the neonatal setting where less branched fetal-type glycogen is dominant $(20,21)$. Glycogen phosphate content is regulated by laforin (22) and evidence from laforin-knockout mouse models suggests that hyperphosphorylated glycogen may promote glycophagy degradation $(10,23)$. Laforin-knockout mice exhibit aggregates of poorly-branched insoluble hyperphosphorylated glycogen associated with elevated expression of the glycophagy receptor protein, Stbd1, in skeletal muscle (10). Thus although the evidence is sparse (and at best anecdotal), these reports suggest that glycophagy may be a process employed under pathophysiological conditions to degrade high molecular weight (possibly $\alpha$-granules), poorly branched and hyperphosphorylated glycogen.

\section{Glycophagosome formation}

Initiation of glycophagy occurs with the formation of the glycophagosome membrane. The overall concepts of phagophore nucleation and phagosome maturation are likely to be similar between autophagy sub-types. In general, in vitro cell culture studies suggest that the phagophore originates from omegasomes; specialized endoplasmic reticulum (ER) subdomains comprised of phosphatidylinositol 3-phosphate-enriched lipid bilayer membranes (24-27). Alternatively, phagophore nucleation has been associated with the trans-golgi network linked with translocation of autophagy-related protein 9 (Atg9) in HeLa and HEK293 mammalian cell lines (28-30). The mitochondria have also been implicated as phagophore membrane donors due to the observation that autophagy-related proteins (e.g. Atg5 and Beclin1) display mitochondrial localization upon autophagy induction $(31,32)$. More recent in vitro work has shown that Atg9-mediated phagophore 
nucleation involves another autophagy protein, Atg2, found on the mitochondria-associated ER membrane (33). It is therefore likely that membrane components are shared between the ER, the mitochondria and the forming phagosome.

Proteomic analysis of hepatic glycogen extracts identified that mitochondrial, lysosomal and ER proteins are associated with glycogen (34). In skeletal muscle, glycogen has been shown to interact with several subcellular compartments, including myofilaments, mitochondria, and the ER (35-37). An ER-glycogenolytic complex has been described in fast-twitch skeletal muscle, aligning phosphorylase-mediated glucose availability with sarcoplasmic reticulum $\mathrm{Ca}^{2+}$ flux ATP demand (36). The glycophagy receptor protein, Stbd1, exhibits binding domains for both the ER and glycogen (38), thus it seems likely that the ER-glycogen complex includes glycophagy mediators, and the concept of an ER-glycogen-phagophore 'hub' has been advanced (39).

\section{Tagging glycogen for glycophagy - the glycophagy 'receptor'}

In parallel with phagophore formation, autophagy is initiated by recruitment of the cellular material to be degraded (cargo) to the autophagosome (Figure 1). Recruitment is mediated by autophagy tagging 'receptor' proteins exhibiting binding sites for both the cargo and Atg8 proteins. Atg8 autophagy proteins include the Lc3 and Gabarap subfamilies which participate in autophagosome maturation (Table 1). Screening of mouse and rat liver glycogen-associated proteins (i.e. the glycogen proteome) identified Stbd1 as a glycogen-binding protein (34). Sequence analysis revealed that Stbd1 exhibits both a carbohydrate (glycogen) binding domain, CBM20 (aa258aa357), and 7 binding sites for Atg8 proteins. Atg8 binding sites (known as Atg8-interacting motifs (AIMs)) are a 4 amino acid sequence, [W/F/Y]-x-x-[I/L/V] (40). Functional AIMs are usually present in intrinsically disordered protein regions and assume tertiary conformation following binding to Atg8 proteins (41). Two of the seven AIMs identified on Stbd1 meet these criteria, spanning amino acids 203-206 and 212-215 (11). Extensive computational analysis of AIMs has shown that the presence of phosphorylatable amino acids located N' terminally to the AIM 
sequence can stabilize its interaction with Atg8 proteins. On Stbd1, both aa203-206 and aa212-215 AIMs exhibit 'upstream' phosphorylated serine residues (Ser175 and Ser211 respectively, Figure 2). Ser175, but not Ser211, has been shown to be phosphorylated by AMPK in a chemical genetic screen (42), suggesting that AMPK may regulate the interaction of Stbd1 with Atg8 proteins via phosphorylating Ser175 near to the aa203-206 AIM.

A role for Stbd1 in glycophagy is further supported by evidence that labeled recombinant Stbd1 co-localizes with the lysosomal-associated membrane protein 1 (Lamp1) in COS cells (10). Furthermore, treatment of HeLa cells with the lysosome inhibitor, bafilomycin, induced accumulation of Stbd1 (38). Interestingly, mutation of the Stbd1 aa203-206 AIM did not affect bafilomycin-induced Stbd1 accumulation (38), suggesting that there may be redundancy between AIMs. In mice lacking the lysosomal glycogen breakdown enzyme, acid $\alpha$-glucosidase (Gaa), glycogen accumulation in autophagolysosomes was dependent on Stbd1 in the liver but not in skeletal muscle (43). These findings support a tissue-specific role for Stbd1 in recruiting glycogen to glycophagy, at least in this experimental context of Gaa knockout. Interestingly, the effect of Stbd1 gene replacement therapy on lysosomal glycogen in Gaa-KO mice was similar with wildtype and aa203-206 AIM mutant Stbd1, but involvement of the other AIM sites has not been investigated (43).

Consistent with the proposal of an 'ER-glycogen-phagophore hub' (39), subcellular localization of Stbd1 aligns with potential sites of early phagosome formation. In COS cells, visualization of Stbd1 was facilitated by genetic addition of an HA-tag and demonstrated that Stbd1 is localized to the ER and the trans-golgi network (38). Comparison with COS cells expressing truncation mutant forms of Stbd1 revealed that the N-terminal hydrophobic region of Stbd1 (aa1-24) is required for ER localization (38). Additionally, it has been demonstrated in HeLa cells that N-myristoylation of Stbd1 plays a role in regulating subcellular localization at the SR and mitochondria (44). As depicted in Figure 2, the glycogen-binding CBM20 domain of Stbd1 has also been shown to 
interact with several glycogen handling proteins such as glycogen synthase, laforin and glycogen debranching enzyme (GDE) (38), but the functional outcomes of these interactions has not been determined.

Although not yet directly investigated, alternative glycophagy 'receptors' may exist. A proteomic analysis of the autophagy interaction network identified that glycogen synthase was an interacting partner of several Atg8 family proteins (12). However in vitro validation experiments showed that glycogen synthase does not bind to Gabarap Atg8 subfamily proteins (12). The glycogen synthase sequence contains several putative AIMs, 1 of which has been investigated in Drosophila. In Drosophila skeletal muscle, glycogen synthase co-localization with Atg8 and sequestration of glycogen to the phagosome appeared to be dependent on the glycogen synthase AIM (45). In silico sequence analysis of common glycogen-related proteins revealed several proteins with putative AIMs (Table 2). These proteins include glycogen synthase, glycogen branching enzyme, glycogen phosphorylase, glycogenin, glycogen debranching enzyme, laforin and malin. Whether these proteins are functional glycophagy receptors is yet to be investigated.

\section{Glycophagosome encapsulation - the glycophagy 'Atg8 partner'}

In the next stage of the autophagy process, the autophagy receptor protein-cargo complex is encapsulated into the forming autophagosome via binding to an Atg8 protein 'partner' (Figure 1). The Atg8 protein family consists of the Lc3 (Lc3a, Lc3b, Lc3c) and Gabarap (Gabarap, Gabarapl1, Gabarapl2) subfamilies, with high sequence similarity (Table 1) (46). In an autophagy network analysis, the glycophagy receptor, Stbd1, was identified to be a candidate interacting protein of all 6 Atg8 family members in HEK293T cells with GST-pulldown assays (12). Additionally a biotinylated peptide screen revealed that the binding affinity of Stbd1 is $\sim 10$-fold higher for Gabarap compared to Lc3b (other Atg8 subfamily members not assessed) (47). Similarly, an Stbd1 yeast 2-hybrid screen identified Gabarap and Gabarapl1, as potential binding partners for Stbd1 (10). Co-expression in vitro validation experiments in COSM9 cells indicated that Stbd1 only 
partially co-localizes with Gabarap while its cellular distribution pattern fully aligns with that of Gabarapl1, producing large perinuclear structures (10). Overexpressed Gabarapl1 localized to the endoplasmic reticulum and trans-golgi network in CHO cells (48), which is similar to the pattern exhibited by overexpressed Stbd1 $(10,38)$. Targeted mutagenesis revealed that the aa203-206 AIM on Stbd1 is essential for Stbd1-Gabarapl1 interaction $(11,49)$ but not for perinuclear localization $(11,38)$ while the opposite was evident for the aa212-215 AIM (11). These findings suggest that the aa212-215 AIM is primarily involved in localization of Stbd1, and the aa203-206 AIM might be the primary target for Gabarapl1 interaction. Furthermore, mutation of the Stbd1 aa203-206 AIM site induces glycogen accumulation in cancer cells in vitro (49), consistent with the contention that Gabarapl1 binding to this Stbd1 AIM is an essential step in glycophagy-mediated degradation of glycogen. Further work is required to understand the complex molecular interplay of these AIMs in mediating glycophagy receptor-Atg8 partner interaction.

Thus Gabarapl1 appears to be the most likely candidate for a glycophagy Atg8 'partner' protein, recruiting Stbd1-bound glycogen into the forming glycophagosome. A degree of redundancy may exist between members of the Atg8 family, particularly in response to various autophagic stimuli and in pathological settings. There is some evidence in cancer cell lines (HeLa) that knockout of LC3B increases lipidation of Gabarapl1 (50). Despite the high sequence similarity between the six Atg8 proteins, emerging evidence suggests that their roles are distinct (46). Computational analysis has revealed that structural differences between Atg8s correspond to a high level of functional specificity (46). In vitro systematic deletion of the Atg8 proteins demonstrated that Gabarap subfamily Atg8s promote autophagy induction via interaction with Unc-51 like autophagy activating kinase 1 (Ulk1), an essential mediator of the early stages of autophagosome biogenesis. In contrast, Lc3 family proteins had an inhibitory effect on Ulk1 activity (50). Starvation, a potent autophagy stimulus, enhances the binding of Ulk1 to Gabarapl1 but has no effect on its interaction with Gabarap or Gabarapl2 (50). In neural stem cells, insulin withdrawal enhanced Ulk1-Gabarapl1 but not Gabarap12 interaction (51). These findings demonstrate that the distinct functionality of the 
Atg8 proteins may be important for their roles in selective autophagy, but to date the knowledge is limited.

Gabarap subfamily Atg8 proteins mediate autophagosome-lysosome fusion in a manner distinct to that of Lc3s, which may have important implications for glycophagy. In mouse embryonic fibroblasts, Lc3 subfamily Atg8 proteins located on the outer autophagosome membrane mediate lysosome fusion in a Stx17-Snap29 dependent fashion while the Gabarap subfamily Atg8 proteins bypass this complex (52). It is so far unclear whether autophagosomes exclusively contain Gabarap or Lc3 Atg8s, but it is likely that subpopulations of Gabarap-dominant phagosomes can bypass the Stx17-Snap29 complex and drive independent autophagosome-lysosome fusion. These contrasting fusion mechanisms may have important implications for delineating glycophagy from other autophagy processes.

\section{Lysosomal glycogenolysis}

Following autophagosome-lysosome fusion, glycogen breakdown in the lysosome is mediated by the $\mathrm{pH}$-dependent glycolytic enzyme, acid $\alpha$-glucosidase (Gaa, Figure 1) (53). In contrast, a dualenzyme action is employed in cytosolic glycogenolysis: glycogen phosphorylase cleaves $\alpha-1,4-$ glycosidic strands in concert with glycogen debranching enzyme which cleaves $\alpha$-1,6-glycosidic branch points. In the lysosome, Gaa has been shown to cleave both $\alpha-1,4$-glycosidic strands and $\alpha$-1,6-glycosidic branch points of the glycogen molecule $(13,14,54)$, however in vitro studies using recombinant human Gaa have shown that Gaa exhibits a 32-fold higher efficiency for cleavage of $\alpha$ 1,4-glycosidic bonds (19). Thus lysosomal glycogen breakdown may favor poorly branched glycogen, consistent with evidence suggesting that Stbd1 glycogen-tagging exhibits higher affinity for polysaccharides with low branching content (10). Additionally, Gaa activity is high in the early postnatal period where less branched fetal-type glycogen is dominant (20). Early studies using fractionated liver homogenates reported that the lysosome is enriched with high molecular weight protein-rich glycogen $(15,55)$ and inhibition of Gaa shifts the relative cellular glycogen content 
towards a low molecular weight profile (56). The physiological relevance of a potential lysosomal 'preference' for protein-rich high molecular weight glycogen is not yet described.

The regulation of lysosomal glycogen breakdown has not been systematically investigated, but some insight can be gleaned from the few studies available. Systemic administration of either cAMP, glucagon, adrenaline or rapamycin in neonatal rats increases hepatic Gaa activity $(20,57-$ 59). Conversely, administration of the $\beta$-adrenergic receptor antagonist, propranolol, decreased Gaa activity (59). Glucose starvation in the postnatal period appears to be a key driver of heightened glycophagy activity in newborn rats as systemic administration of glucose during this period abolished the postnatal glycophagy response (59). In mouse embryonic fibroblasts mTOR activation via amino acid starvation was associated with increased glycophagy activity (60). These rodent studies suggest that lysosomal glycogen degradation is regulated by energy signaling pathways involving cAMP and mTOR, at least in the postnatal period of heightened glycophagy activity.

Several aspects of lysosomal glycogen degradation have received very little or no attention in the literature. In particular, there is limited knowledge of the mechanism of glucose liberation from the lysosome. There has been some indication that the Glut8 transporter may be involved in lysosomal glucose release, but conflicting reports exist. Glut8 exhibits an endosomal/lysosomal targeting sequence $(61,62)$, and in Glut8-stably expressing CHO and MB231 cells, Glut8 colocalized with the lysosomal protein Lamp1 and was detected in lysosomal membranes $(61,63)$. Furthermore, inhibition of the lysosome with chloroquine or bafilomycin resulted in an accumulation of Glut8 (63). However not all reports support a role for Glut8 in the lysosome. In PC12 neuronal cells, Glut8 colocalized with markers for the endoplasmic reticulum but not the lysosome (64). Other studies show that Glut8 is an insulin-sensitive plasma membrane transporter which participates in cellular glucose uptake in the heart and other tissues $(65,66)$. Yet an in vitro study screening gainand loss-of-function of Glut8, showed that knockdown of Glut8 does not influence 2-deoxyglucose uptake (63). Thus whether Glut8 is operational as a lysosomal glucose transporter and has a role in 
glycophagy has not been fully elucidated. There is some evidence that a lysosomal efflux permease, Spin, may also be linked with lysosomal glucose release. Knockout of Spin in both rat kidney epithelial cultured cells and Drosophila resulted in enlarged, carbohydrate-rich lysosomes (67). Additionally, knockout of the predicted lysosomal sugar carrier, the benchwarmer gene, in Drosophila induced carbohyielsendrate accumulation and lysosomal impairment (68). These findings provide new opportunities for investigation into the mechanism of lysosomal glucose transport in health and disease settings.

Inherited Gaa deficiency causes a glycogen storage disorder, Pompe disease (69), characterized by lysosomal glycogen accumulation in nerve and muscle cells, leading to progressive cardiac and respiratory complications and premature death $(70,71)$. The phenotypic severity of inherited Gaa mutations exemplify that glycophagy and lysosomal degradation of glycogen are essential processes to maintain cellular function. Beyond inherited glycogen storage disorders, there is some indication that disturbances in glycophagy may play a role in disease settings involving energy stress $(4,72$, 73). Autophagy has become an elusive target for disease intervention $(74,75)$, and modulating selective-autophagy processes may be a more viable therapeutic strategy. Given that glycophagy is a fundamental cellular process positioned at the interface of autophagy and metabolism, it is likely to be an important target for metabolic disease intervention (76). Thus advancing knowledge of the mechanisms and regulation of lysosomal glycogen breakdown is an important priority.

\section{Conclusion}

Recent advances in understanding selective-autophagy pathways have opened up new areas of investigation in relation to the metabolism of key metabolic substrates, including glycogen. Glycophagy has emerged as a non-canonical pathway of glycogen flux, and its' role in mediating physiological carbohydrate metabolism is yet to be fully elucidated. The field of glycophagy research is at an early evolutionary stage, and the available reports identifying the protein machinery involved provide new leads for investigative pursuit. The current state of evidence 
suggests that specific glycophagy protein machinery is employed, with Stbd1 as the glycophagy 'receptor', Gabarapl1 as the 'Atg8 partner' autophagosome protein, and acid $\alpha$-glucosidase as the lysosomal glycogen degradation enzyme. Additional/ alternative protein mediators may emerge with future research focus. Opportunities exist for addressing major knowledge gaps in understanding the role of glycophagy in health and disease, and identifying the key regulatory signals and specific protein intermediaries is an important next step. Given the challenges of translating autophagy therapeutics to clinical application, highly specific interventions targeting glycophagy may yield valuable outcomes in several disease contexts.

\section{Conflict of interest}

The authors have no conflicts of interest to declare. 


\section{References}

1. Tanida, I. (2011) Autophagy basics. Microbiol Immunol. 55, 1-11

2. Johansen, T., and Lamark, T. (2020) Selective Autophagy: ATG8 Family Proteins, LIR Motifs and Cargo Receptors. J Mol Biol. 432, 80-103

3. Gatica, D., Lahiri, V., and Klionsky, D. J. (2018) Cargo recognition and degradation by selective autophagy. Nat Cell Biol. 20, 233-242

4. Delbridge, L. M. D., Mellor, K. M., Taylor, D. J. R., and Gottlieb, R. A. (2015) Myocardial autophagic energy stress responses - macroautophagy, mitophagy, and glycophagy. American Journal of Physiology-Heart and Circulatory Physiology. 308, H1194-H1204

5. Stolz, A., Ernst, A., and Dikic, I. (2014) Cargo recognition and trafficking in selective autophagy. Nat Cell Biol. 16, 495-501

6. Prats, C., Graham, T. E., and Shearer, J. (2018) The dynamic life of the glycogen granule. $J$. Biol. Chem. 293, 7089-7098

7. Chandramouli, C., Varma, U., Stevens, E. M., Xiao, R.-P., Stapleton, D. I., Mellor, K. M., and Delbridge, L. M. D. (2015) Myocardial glycogen dynamics: new perspectives on disease mechanisms. Clin. Exp. Pharmacol. Physiol. 42, 415-425

8. Mellor, K. M., Varma, U., Stapleton, D. I., and Delbridge, L. M. D. (2014) Cardiomyocyte glycophagy is regulated by insulin and exposure to high extracellular glucose. American Journal of Physiology-Heart and Circulatory Physiology. 306, H1240-H1245

9. Reichelt, M. E., Mellor, K. M., Curl, C. L., Stapleton, D., and Delbridge, L. M. D. (2013) Myocardial glycophagy - a specific glycogen handling response to metabolic stress is accentuated in the female heart. J. Mol. Cell. Cardiol. 65, 67-75

10. Jiang, S., Heller, B., Tagliabracci, V. S., Zhai, L., Irimia, J. M., DePaoli-Roach, A. A., Wells, C. D., Skurat, A. V., and Roach, P. J. (2010) Starch Binding Domain-containing Protein 1/Genethonin 1 Is a Novel Participant in Glycogen Metabolism. J Biol Chem. 285, 3496034971

11. Jiang, S., Wells, C. D., and Roach, P. J. (2011) Starch-binding domain-containing protein 1 (Stbd1) and glycogen metabolism: Identification of the Atg8 family interacting motif (AIM) in Stbd1 required for interaction with GABARAPL1. Biochem Biophys Res Commun. 413, 420 425

12. Behrends, C., Sowa, M. E., Gygi, S. P., and Harper, J. W. (2010) Network organization of the human autophagy system. Nature. 466, 68-76

13. Palmer, T. N. (1971) The substrate specificity of acid $\alpha$-glucosidase from rabbit muscle. Biochem J. 124, 701-711

14. Tashiro, K., Iwamasa, T., Kato, H., Ogata, S., and Anai, M. (1986) Purification and Characterization of Two Components of Acid $\alpha$-Glucosidase from Pig Liver 1. The Journal of Biochemistry. 99, 693-701

15. Geddes, R., and Stratton, G. C. (1977) The influence of lysosomes on glycogen metabolism. Biochem J. 163, 193-200

16. Roach, P. J., Depaoli-Roach, A. A., Hurley, T. D., and Tagliabracci, V. S. (2012) Glycogen and its metabolism: some new developments and old themes. Biochem. J. 441, 763-787

17. Ryu, J.-H., Drain, J., Kim, J. H., McGee, S., Gray-Weale, A., Waddington, L., Parker, G. J., Hargreaves, M., Yoo, S.-H., and Stapleton, D. (2009) Comparative structural analyses of purified glycogen particles from rat liver, human skeletal muscle and commercial preparations. Int J Biol Macromol. 45, 478-482

18. Arunachalam, B., Phan, U. T., Geuze, H. J., and Cresswell, P. (2000) Enzymatic reduction of disulfide bonds in lysosomes: characterization of a gamma-interferon-inducible lysosomal thiol reductase (GILT). Proc Natl Acad Sci U S A. 97, 745-750

19. Roig-Zamboni, V., Cobucci-Ponzano, B., Iacono, R., Ferrara, M. C., Germany, S., Bourne, Y., Parenti, G., Moracci, M., and Sulzenbacher, G. (2017) Structure of human lysosomal acid $\alpha$ glucosidase-a guide for the treatment of Pompe disease. Nat Commun. 8, 111 
20. Kondomerkos, D. J., Kalamidas, S. A., Kotoulas, O. B., and Hann, A. C. (2005) Glycogen autophagy in the liver and heart of newborn rats. The effects of glucagon, adrenalin or rapamycin. Histol Histopathol. 20, 689-696

21. Iwamasa, T., Tsuru, T., Hamada, T., and Takeuchi, T. (1980) Physicochemical and ultrastructural studies on glycogenosomes in newborn rat hepatocytes. Pathol Res Pract. 167, 363-373

22. Gentry, M. S., Guinovart, J. J., Minassian, B. A., Roach, P. J., and Serratosa, J. M. (2018) Lafora disease offers a unique window into neuronal glycogen metabolism. J Biol Chem. 293, $7117-7125$

23. Tagliabracci, V. S., Turnbull, J., Wang, W., Girard, J.-M., Zhao, X., Skurat, A. V., DelgadoEscueta, A. V., Minassian, B. A., Depaoli-Roach, A. A., and Roach, P. J. (2007) Laforin is a glycogen phosphatase, deficiency of which leads to elevated phosphorylation of glycogen in vivo. Proc Natl Acad Sci US A. 104, 19262-19266

24. Axe, E. L., Walker, S. A., Manifava, M., Chandra, P., Roderick, H. L., Habermann, A., Griffiths, G., and Ktistakis, N. T. (2008) Autophagosome formation from membrane compartments enriched in phosphatidylinositol 3-phosphate and dynamically connected to the endoplasmic reticulum. $J$ Cell Biol. 182, 685-701

25. Hayashi-Nishino, M., Fujita, N., Noda, T., Yamaguchi, A., Yoshimori, T., and Yamamoto, A. (2009) A subdomain of the endoplasmic reticulum forms a cradle for autophagosome formation. Nat Cell Biol. 11, 1433-1437

26. Uemura, T., Yamamoto, M., Kametaka, A., Sou, Y., Yabashi, A., Yamada, A., Annoh, H., Kametaka, S., Komatsu, M., and Waguri, S. (2014) A cluster of thin tubular structures mediates transformation of the endoplasmic reticulum to autophagic isolation membrane. $\mathrm{Mol}$ Cell Biol. 34, 1695-1706

27. Ylä-Anttila, P., Vihinen, H., Jokitalo, E., and Eskelinen, E.-L. (2009) 3D tomography reveals connections between the phagophore and endoplasmic reticulum. Autophagy. 5, 1180-1185

28. Orsi, A., Razi, M., Dooley, H. C., Robinson, D., Weston, A. E., Collinson, L. M., and Tooze, S. A. (2012) Dynamic and transient interactions of Atg9 with autophagosomes, but not membrane integration, are required for autophagy. Mol Biol Cell. 23, 1860-1873

29. Takahashi, Y., Meyerkord, C. L., Hori, T., Runkle, K., Fox, T. E., Kester, M., Loughran, T. P., and Wang, H.-G. (2011) Bif-1 regulates Atg9 trafficking by mediating the fission of Golgi membranes during autophagy. Autophagy. 7, 61-73

30. Takahashi, Y., Tsotakos, N., Liu, Y., Young, M. M., Serfass, J., Tang, Z., Abraham, T., and Wang, H.-G. (2016) The Bif-1-Dynamin 2 membrane fission machinery regulates Atg9containing vesicle generation at the Rab11-positive reservoirs. Oncotarget. 7, 20855-20868

31. Hailey, D. W., Rambold, A. S., Satpute-Krishnan, P., Mitra, K., Sougrat, R., Kim, P. K., and Lippincott-Schwartz, J. (2010) Mitochondria supply membranes for autophagosome biogenesis during starvation. Cell. 141, 656-667

32. Tooze, S. A., and Yoshimori, T. (2010) The origin of the autophagosomal membrane. Nat. Cell Biol. 12, 831-835

33. Tang, Z., Takahashi, Y., He, H., Hattori, T., Chen, C., Liang, X., Chen, H., Young, M. M., and Wang, H.-G. (2019) TOM40 Targets Atg2 to Mitochondria-Associated ER Membranes for Phagophore Expansion. Cell Rep. 28, 1744-1757.e5

34. Stapleton, D., Nelson, C., Parsawar, K., McClain, D., Gilbert-Wilson, R., Barker, E., Rudd, B., Brown, K., Hendrix, W., O’Donnell, P., and Parker, G. (2010) Analysis of hepatic glycogenassociated proteins. Proteomics. 10, 2320-2329

35. Wanson, J. C., and Drochmans, P. (1972) Role of the sarcoplasmic reticulum in glycogen metabolism. Binding of phosphorylase, phosphorylase kinase, and primer complexes to the sarcovesicles of rabbit skeletal muscle. J Cell Biol. 54, 206-224

36. Entman, M. L., Keslensky, S. S., Chu, A., and Van Winkle, W. B. (1980) The sarcoplasmic reticulum-glycogenolytic complex in mammalian fast twitch skeletal muscle. Proposed in vitro counterpart of the contraction-activated glycogenolytic pool. J Biol Chem. 255, 6245-6252 
37. Nielsen, J., Schrøder, H. D., Rix, C. G., and Ortenblad, N. (2009) Distinct effects of subcellular glycogen localization on tetanic relaxation time and endurance in mechanically skinned rat skeletal muscle fibres. J Physiol. 587, 3679-3690

38. Zhu, Y., Zhang, M., Kelly, A. R., and Cheng, A. (2014) The carbohydrate-binding domain of overexpressed STBD1 is important for its stability and protein-protein interactions. Biosci Rep. 10.1042/BSR20140053

39. Mandl, J., and Bánhegyi, G. (2018) The ER - Glycogen Particle - Phagophore Triangle: A Hub Connecting Glycogenolysis and Glycophagy? Pathol Oncol Res. 24, 821-826

40. Noda, N. N., Ohsumi, Y., and Inagaki, F. (2010) Atg8-family interacting motif crucial for selective autophagy. FEBS Lett. 584, 1379-1385

41. Popelka, H., and Klionsky, D. J. (2015) Analysis of the native conformation of the LIR/AIM motif in the Atg8/LC3/GABARAP-binding proteins. Autophagy. 11, 2153-2159

42. Ducommun, S., Deak, M., Zeigerer, A., Göransson, O., Seitz, S., Collodet, C., Madsen, A. B., Jensen, T. E., Viollet, B., Foretz, M., Gut, P., Sumpton, D., and Sakamoto, K. (2019) Chemical genetic screen identifies Gapex-5/GAPVD1 and STBD1 as novel AMPK substrates. Cell Signal. 57, 45-57

43. Sun, T., Yi, H., Yang, C., Kishnani, P. S., and Sun, B. (2016) Starch Binding Domaincontaining Protein 1 Plays a Dominant Role in Glycogen Transport to Lysosomes in Liver. $J$ Biol Chem. 291, 16479-16484

44. Demetriadou, A., Morales-Sanfrutos, J., Nearchou, M., Baba, O., Kyriacou, K., Tate, E. W., Drousiotou, A., and Petrou, P. P. (2017) Mouse Stbd1 is N-myristoylated and affects ERmitochondria association and mitochondrial morphology. J Cell Sci. 130, 903-915

45. Zirin, J., Nieuwenhuis, J., and Perrimon, N. (2013) Role of autophagy in glycogen breakdown and its relevance to chloroquine myopathy. PLoS Biol. 11, e1001708

46. Jatana, N., Ascher, D. B., Pires, D. E. V., Gokhale, R. S., and Thukral, L. (2020) Human LC3 and GABARAP subfamily members achieve functional specificity via specific structural modulations. Autophagy. 16, 239-255

47. Rogov, V. V., Stolz, A., Ravichandran, A. C., Rios-Szwed, D. O., Suzuki, H., Kniss, A., Löhr, F., Wakatsuki, S., Dötsch, V., Dikic, I., Dobson, R. C., and McEwan, D. G. (2017) Structural and functional analysis of the GABARAP interaction motif (GIM). EMBO Rep. 18, 13821396

48. Chen, C., Li, J.-G., Chen, Y., Huang, P., Wang, Y., and Liu-Chen, L.-Y. (2006) GEC1 interacts with the kappa opioid receptor and enhances expression of the receptor. J Biol Chem. 281, 7983-7993

49. Han, Z., Zhang, W., Ning, W., Wang, C., Deng, W., Li, Z., Shang, Z., Shen, X., Liu, X., Baba, O., Morita, T., Chen, L., Xue, Y., and Jia, D. (2021) Model-based analysis uncovers mutations altering autophagy selectivity in human cancer. Nat Commun. 12, 3258

50. Grunwald, D. S., Otto, N. M., Park, J.-M., Song, D., and Kim, D.-H. (2020) GABARAPs and LC3s have opposite roles in regulating ULK1 for autophagy induction. Autophagy. 16, 600614

51. Ryu, H. Y., Kim, L. E., Jeong, H., Yeo, B. K., Lee, J.-W., Nam, H., Ha, S., An, H.-K., Park, H., Jung, S., Chung, K. M., Kim, J., Lee, B.-H., Cheong, H., Kim, E.-K., and Yu, S.-W. (2021) GSK3B induces autophagy by phosphorylating ULK1. Exp Mol Med. 53, 369-383

52. Ebner, P., Poetsch, I., Deszcz, L., Hoffmann, T., Zuber, J., and Ikeda, F. (2018) The IAP family member BRUCE regulates autophagosome-lysosome fusion. Nat Commun. 9, 599

53. Chambers, J. P., and Williams, J. C. (1983) Acid alpha-glucosidase from human heart. Enzyme. 29, 109-119

54. Onodera, S., Matsui, H., and Chibat, S. (1994) Substrate Specificity and Subsite Affinities of Rabbit Liver Acid $\alpha$-Glucosidase1. The Journal of Biochemistry. 116, 7-11

55. Geddes, R., Harvey, J. D., and Wills, P. R. (1977) The molecular size and shape of liver glycogen. Biochem J. 163, 201-209 
56. Geddes, R., Otter, D. E., Scott, G. K., and Taylor, J. A. (1983) Disturbance of lysosomal glycogen metabolism by liposomal anti-alpha-glucosidase and some anti-inflammatory drugs. Biochem J. 212, 99-103

57. Kondomerkos, D. J., Kalamidas, S. A., and Kotoulas, O. B. (2004) An electron microscopic and biochemical study of the effects of glucagon on glycogen autophagy in the liver and heart of newborn rats. Microscopy Research and Technique. 63, 87-93

58. Kotoulas, O. B. (1986) The effects of cyclic 3',5'-AMP on the lysosomes of newborn rat hepatocytes. J Ultrastruct Mol Struct Res. 97, 210-215

59. Kalamidas, S. A., and Kotoulas, O. B. (1999) The degradation of glycogen in the lysosomes of newborn rat hepatocytes: glycogen-, maltose- and isomaltose-hydrolyzing acid alpha glucosidase activities in liver. Histol Histopathol. 14, 23-30

60. Pal, R., Xiong, Y., and Sardiello, M. (2019) Abnormal glycogen storage in tuberous sclerosis complex caused by impairment of mTORC1-dependent and -independent signaling pathways. Proc Natl Acad Sci U S A. 116, 2977-2986

61. Augustin, R., Riley, J., and Moley, K. H. (2005) GLUT8 contains a [DE]XXXL[LI] sorting motif and localizes to a late endosomal/lysosomal compartment. Traffic. 6, 1196-1212

62. Diril, M. K., Schmidt, S., Krauss, M., Gawlik, V., Joost, H.-G., Schürmann, A., Haucke, V., and Augustin, R. (2009) Lysosomal localization of GLUT8 in the testis--the EXXXLL motif of GLUT8 is sufficient for its intracellular sorting via AP1- and AP2-mediated interaction. FEBS J. 276, 3729-3743

63. Alexander, C. M., Martin, J. A., Oxman, E., Kasza, I., Senn, K. A., and Dvinge, H. (2020) Alternative Splicing and Cleavage of GLUT8. Mol Cell Biol. 10.1128/MCB.00480-20

64. Widmer, M., Uldry, M., and Thorens, B. (2005) GLUT8 subcellular localization and absence of translocation to the plasma membrane in PC12 cells and hippocampal neurons. Endocrinology. 146, 4727-4736

65. Carayannopoulos, M. O., Chi, M. M., Cui, Y., Pingsterhaus, J. M., McKnight, R. A., Mueckler, M., Devaskar, S. U., and Moley, K. H. (2000) GLUT8 is a glucose transporter responsible for insulin-stimulated glucose uptake in the blastocyst. Proc Natl Acad Sci US A. 97, 7313-7318

66. Maria, Z., Campolo, A. R., and Lacombe, V. A. (2015) Diabetes Alters the Expression and Translocation of the Insulin-Sensitive Glucose Transporters 4 and 8 in the Atria. PLoS One. 10, e0146033

67. Rong, Y., McPhee, C. K., McPhee, C., Deng, S., Huang, L., Chen, L., Liu, M., Tracy, K., Baehrecke, E. H., Baehreck, E. H., Yu, L., and Lenardo, M. J. (2011) Spinster is required for autophagic lysosome reformation and mTOR reactivation following starvation. Proc Natl Acad Sci U S A. 108, 7826-7831

68. Dermaut, B., Norga, K. K., Kania, A., Verstreken, P., Pan, H., Zhou, Y., Callaerts, P., and Bellen, H. J. (2005) Aberrant lysosomal carbohydrate storage accompanies endocytic defects and neurodegeneration in Drosophila benchwarmer. J Cell Biol. 170, 127-139

69. Chan, J., Desai, A. K., Kazi, Z. B., Corey, K., Austin, S., Hobson-Webb, L. D., Case, L. E., Jones, H. N., and Kishnani, P. S. (2017) The emerging phenotype of late-onset Pompe disease: A systematic literature review. Mol Genet Metab. 120, 163-172

70. Bulkley, B. H., and Hutchins, G. M. (1978) Pompe's disease presenting as hypertrophic myocardiopathy with Wolff-Parkinson-White syndrome. Am Heart J. 96, 246-252

71. Francesconi, M., and Auff, E. (1982) Cardiac arrhythmias and the adult form of type II glycogenosis. N Engl J Med. 306, 937-938

72. Delbridge, L. M. D., Mellor, K. M., Taylor, D. J., and Gottlieb, R. A. (2017) Myocardial stress and autophagy: mechanisms and potential therapies. Nat Rev Cardiol. 14, 412-425

73. Daniels, L. J., Varma, U., Annandale, M., Chan, E., Mellor, K. M., and Delbridge, L. M. D. (2019) Myocardial Energy Stress, Autophagy Induction, and Cardiomyocyte Functional Responses. Antioxid Redox Signal. 31, 472-486 
74. Mellor, K. M., Reichelt, M. E., and Delbridge, L. M. D. (2011) Autophagy anomalies in the diabetic myocardium. Autophagy. 7, 1263-1267

75. Condello, M., Pellegrini, E., Caraglia, M., and Meschini, S. (2019) Targeting Autophagy to Overcome Human Diseases. Int J Mol Sci. 10.3390/ijms20030725

76. Zhao, H., Tang, M., Liu, M., and Chen, L. (2018) Glycophagy: An emerging target in pathology. Clin Chim Acta. 484, 298-303 
Table 1. Human Atg8 family protein sequence similarity.

\begin{tabular}{|c|c|c|c|}
\hline $\begin{array}{l}\text { Atg8 } \\
\text { protein }\end{array}$ & Sequence & $\begin{array}{c}\text { Sequence } \\
\text { similarity to } \\
\text { Lc3a }\end{array}$ & $\begin{array}{l}\text { Sequence } \\
\text { similarity to } \\
\text { Gabarap }\end{array}$ \\
\hline \multicolumn{4}{|c|}{ Lc3 subfamily } \\
\hline Lc3a & $\begin{array}{l}\text { MPSDRPFKQRRSFADRCKEVQQIRDQHPSKIPVIIERYKG } \\
\text { EKQLPVLDKTKFLVPDHVNMSELVKIIRRRLQLNPTQAFFL } \\
\text { LVNQHSMVSVSTPIADIYEQEKDEDGFLYMVYASQTFG } \\
\text { F }\end{array}$ & & $31.6 \%$ \\
\hline Lc3b & $\begin{array}{l}\text { MPSEKTFKQRRTFEQRVEDVRLIREQHPTKIPVIIERYKGE } \\
\text { KQLPVLDKTKFLVPDHVNMSELIKIIRRRLQLNANQAFFLL } \\
\text { VNGHSMVSVSTPISEVYESEKDEDGFLYMVYASQETFGM } \\
\text { KLSV }\end{array}$ & $82.5 \%$ & $31.0 \%$ \\
\hline Lc3c & $\begin{array}{l}\text { MPPPQKIPSVRPFKQRKSLAIRQEEVAGIRAKFPNKIPVVV } \\
\text { ERYPRETFLPPLDKTKFLVPQELTMTQFLSIIRSRMVLRAT } \\
\text { EAFYLLVNNKSLVSMSATMAEIYRDYKDEDGFVYMTYAS } \\
\text { QETFGCLESAAPRDGSSLEDRPCNPL }\end{array}$ & $59.2 \%$ & $39.3 \%$ \\
\hline \multicolumn{4}{|c|}{ Gabarap subfamily } \\
\hline Gabarap & $\begin{array}{l}\text { MKFVYKEEHPFEKRRSEGEKIRKKYPDRVPVIVEKAPKAR } \\
\text { IGDLDKKKYLVPSDLTVGQFYFLIRKRIHLRAEDALFFFVN } \\
\text { NVIPPTSATMGQLYQEHHEEDFFLYIAYSDESVYGL }\end{array}$ & $31.6 \%$ & \\
\hline Gabarapl1 & $\begin{array}{l}\text { MKFQYKEDHPFEYRKKEGEKIRKKYPDRVPVIVEKAPKA } \\
\text { RVPDLDKRKYLVPSDLTVGQFYFLIRKRIHLRPEDALFFFV } \\
\text { NNTIPPTSATMGQLYEDNHEEDYFLYVAYSDESVYGK }\end{array}$ & $34.2 \%$ & $87.1 \%$ \\
\hline Gabarapl2 & $\begin{array}{l}\text { MKWMFKEDHSLEHRCVESAKIRAKYPDRVPVIVEKVSGS } \\
\text { QIVDIDKRKYLVPSDITVAQFMWIIRKRIQLPSEKAIFLFVD } \\
\text { KTVPQSSLTMGQLYEKEKDEDGFLYVAYSGENTFGF }\end{array}$ & $41.7 \%$ & $57.8 \%$ \\
\hline
\end{tabular}


Table 2. Atg8-interacting motifs (AIMs) identified in human glycogen-related proteins in silico.

\begin{tabular}{|c|c|c|c|c|c|}
\hline \multirow[b]{2}{*}{ Protein } & \multirow[b]{2}{*}{ Gene } & \multirow{2}{*}{$\begin{array}{l}\text { Uniprot } \\
\text { identifier }\end{array}$} & \multicolumn{3}{|c|}{ Atg8-interacting motifs } \\
\hline & & & $\begin{array}{l}\text { No. of Lc3-interacting } \\
\text { regions (LIR) }\end{array}$ & $\begin{array}{l}\text { No. of 'strict' Gabarap- } \\
\text { interacting motifs (GIM) }\end{array}$ & $\begin{array}{l}\text { No. of 'loose' Gabarap- } \\
\text { interacting motifs (GIM) }\end{array}$ \\
\hline Starch-binding domain-containing protein 1 & STBD1 & 095210 & 10 & 2 & 5 \\
\hline Glycogen synthase 1 & GYS1 & P13807 & 12 & 0 & 2 \\
\hline Glycogen synthase 2 & GYS2 & P54840 & 12 & 0 & 2 \\
\hline Glycogen branching enzyme & AGL & Q04446 & 14 & 0 & 3 \\
\hline Glycogen phosphorylase muscle & PYGM & P11217 & 10 & 0 & 1 \\
\hline Glycogen phosphorylase liver & PYGL & P06737 & 11 & 0 & 1 \\
\hline Glycogen phosphorylase brain & PYGB & P11216 & 11 & 0 & 1 \\
\hline Glycogenin 1 & GYG1 & P46976 & 9 & 0 & 2 \\
\hline Glycogenin 2 & GYG2 & O15488 & 7 & 0 & 3 \\
\hline Glycogen debranching enzyme & GDE & P35573 & 39 & 1 & 4 \\
\hline Laforin & EPM2A & O95278 & 6 & 0 & 3 \\
\hline Malin & NHLRC1 & Q6VVB1 & 2 & 0 & 0 \\
\hline
\end{tabular}

LC3 interacting motif: [WFY]-x-x-[ILV] (74). GABARAP interacting motif (strict): [WF]-[VI]-x-V (47). GABARAP interacting motif (loose): [WF]$\mathrm{X}-\mathrm{X}-\mathrm{V}$ (47). The glycogen-related proteins were screened for LC3- and Gabarap-interacting motifs using Expasy ScanProsite tool (https://prosite.expasy.org/scanprosite/).

. 


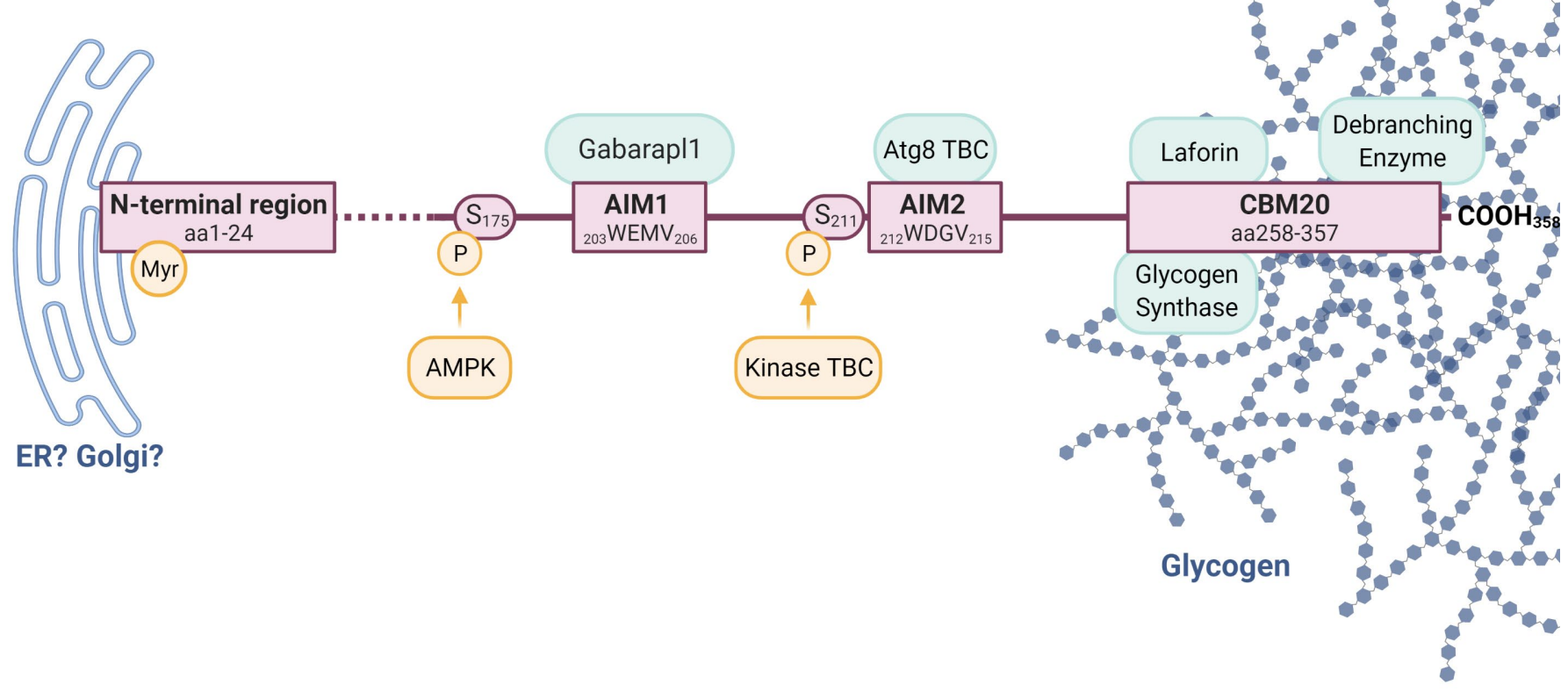

Figure 2. STBD1 protein domains, interacting proteins and post-translational modifications. The glycogenbinding protein, STBD1 (purple), contains 2 putative functional Atg8-interacting motifs (AIM) potentially regulated by phosphorylation of serine $(\mathrm{S})$ residues located $\mathrm{N}$-terminally to the AIM site. The carbohydrate binding domain (CBM20) binds to glycogen. Evidence suggests that Gabarapl1 is the Atg8 family protein that binds to AIM1. AMP-activated protein kinase (AMPK) has been shown to phosphorylate Ser175 and may be involved in regulating the AIM1-Gabarapl1 interaction. Several glycogen-related proteins bind to the CBM20 site including laforin, glycogen debranching enzyme and glycogen synthase. Myristoylation (Myr) of the Nterminus may play a role in determining subcellular location, and the endoplasmic reticulum (ER) and/or the golgi apparatus may be the site of STBD1 localization and phagophore nucleation. 